\title{
Airway loss during inhalation induction of anesthesia with sevoflurane in a pediatric patient with laryngeal papillomatosis: A case report
}

\author{
XIAO HU and XIA SHEN \\ Department of Anesthesiology, The Eye, Ear, Nose and Throat Hospital of Fudan University, \\ Shanghai Medical College of Fudan University, Shanghai 200031, P.R. China
}

Received September 11, 2014; Accepted September 14, 2015

DOI: 10.3892/etm.2015.2805

\begin{abstract}
The aim of the present study was to report a failed inhalation induction of anesthesia with sevofluran in a pediatric patient with grade III laryngeal obstruction caused by laryngeal papillomatosis. Inhalation induction is recommended for the induction of anesthesia in pediatric patients. In the present case, due to the respiratory depression properties of the anesthetics, the partial airway obstruction developed into complete airway obstruction, followed by cardiac arrest. The patient survived after the surgeon managed to reconvert the complete airway obstruction into partial airway obstruction through the removal of part of the laryngeal papillomas. In conclusion, the reaction of the patient in the present case to sevoflurane inhalation induction suggests that this technique is not recommended for patients with pre-existing severe airway obstruction. In such cases, special consideration and preparation is required.
\end{abstract}

\section{Introduction}

Laryngeal papillomas are benign epithelial tumors that are caused by infection with the human papilloma virus. Previous studies have estimated that the incidence rate of these lesions is 4.3 per 100,000 children and 1.8 per 100,000 adults $(1,2)$. Currently, no cure exists for recurrent pediatric laryngeal papillomatosis. The primary management strategy is surgical removal of the tumor under a laryngoscope at frequent intervals to relieve symptoms of airway obstruction (3). A spontaneous ventilation technique is preferred in children with upper airway obstruction, since

Correspondence to: Dr Xia Shen, Department of Anesthesiology, The Eye, Ear, Nose and Throat Hospital of Fudan University, Shaghai Medical College of Fudan Universtiy, 83 Fenyang Road, Shanghai 200031, P.R. China

E-mail: zlsx@yahoo.com

Key words: airway management, airway complication, induction, laryngeal papillomatosis, pediatric this technique maintains a degree of muscle tone and helps to maintain gas exchange (4). Sevoflurane is widely used for anesthesia induction in pediatric population (5). However, airway loss may occur during inhalation induction of anesthesia in pediatric patients with pre-existing severe airway compromise (6).

The present study reported the case of a 22-month old pediatric patient with grade III laryngeal obstruction, which developed into complete airway obstruction and led to cardiac arrest during sevoflurane inhalation induction.

\section{Case report}

A 22-month-old boy (admitted on December 9th, 2013; weight, $14 \mathrm{~kg}$ ) was scheduled for an emergency laryngeal papillomatosis resection at the Eye, Ear, Nose and Throat Hospital of Fudan University (Shanghai, China) to relieve airway obstruction. The patient had previously undergone three more uneventful laryngeal papillomatosis resections at the age of 16, 18, 19 and 20 months. A lateral neck X-ray scan showed the presence of recurrent laryngeal papillomatosis at the glottic and subglottic levels (Fig. 1).

The patient presented grade III laryngeal obstruction (6) with the following clinical indications: Marked inspiratory dyspnea and laryngeal stridor, evidence of a concave in the suprasternal notch and supraclavicular fossa, adversely affected sleeping and eating habits, and dysphoria. Informed consent was obtained from the legal guardians of the patient prior to anesthesia and surgery. The patient did not receive any premedication for potential respiratory depression.

Inhalation induction of anesthesia with $8 \%$ sevoflurane in $6 \mathrm{l} / \mathrm{min}$ oxygen was performed prior to the surgery. The vital signs and a capnogram were found to be normal. After 5 min, the patient presented with abnormal breathing and $\mathrm{CO}_{2}$ expiration recorded by the capnogram was reduced. The anesthesiologist inserted an oral airway and used the jaw-thrust maneuver to open the airway as much as possible; however, the technique was unsuccessful in opening the airway. Subsequently, the capnogram disappeared, the peripheral capillary oxygen saturation of the patient decreased and manual ventilation was rendered impossible. Sevoflurane was discontinued and the anesthesiologist attempted to intubate 


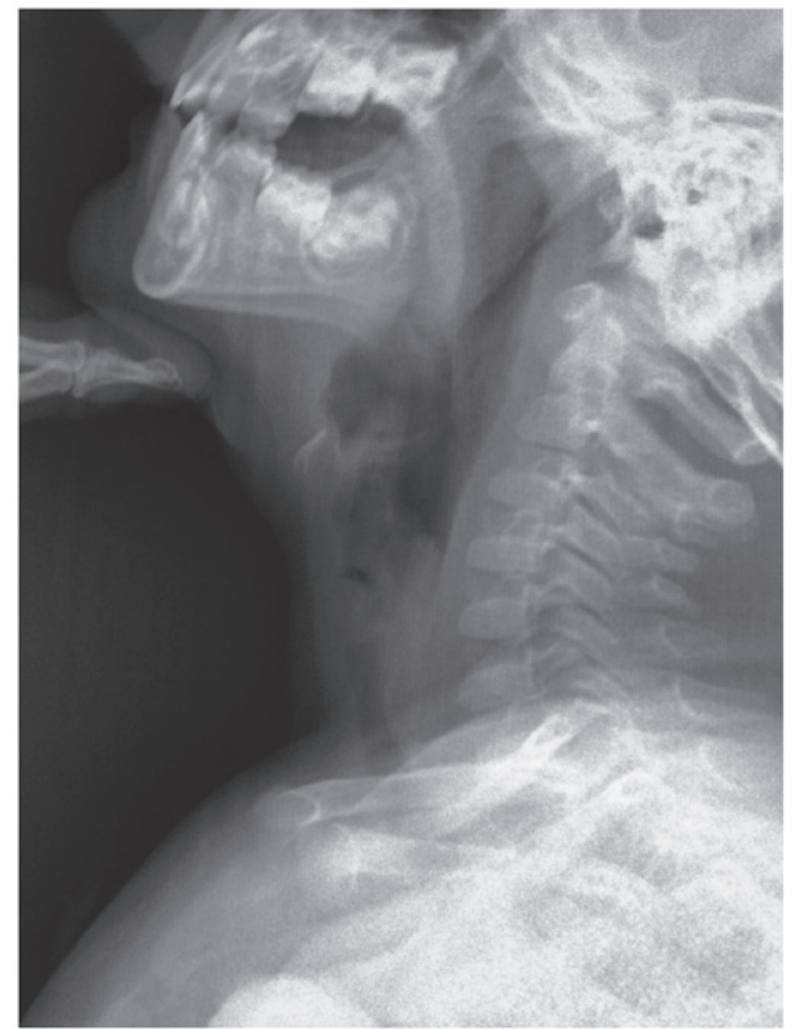

Figure 1. Neck X-ray scan, showing heavy soft tissue at the glottic and subglottic areas.

a 3-mm (inner diameter) tracheal tube with a stylet via direct laryngoscopy, but the vocal cord or epiglottis could not be identified. The patient did not wake up or resume spontaneous respiration. The laryngeal inlet was obstructed by papillomatosis, thus the surgeons attempted to open the airway by removing part of the papillomatosis. Subsequently, the child suffered a cardiac arrest and cardiopulmonary resuscitation (CPR) was performed. A size 2 laryngeal mask airway was used, but manual ventilation remained impossible. A cricothyrotomy was then performed, followed by a tracheotomy, which were unsuccessful. Finally, a tracheal intubation was reattempted, which was succeeful. The time interval between CPR and successful tracheal intubation was $10 \mathrm{~min}$.

The surgeon then completed the tracheotomy and resection of the laryngeal papillomatosis tumor under sevoflurane at 1 minimum alveolar concentration (MAC) and remifentanil anesthesia at an infusion rate of 0.05-0.1 $\mu \mathrm{g} \mathrm{x} \mathrm{kg} \mathrm{min}{ }^{-1}$. Following surgery, a $1.5-\mathrm{mm}$ (inner diameter) tracheostomy tube was placed and the patient was transferred to the Intensive Care Unit for recovery under dexmedetomidine sedation (a bolus of $1 \mu \mathrm{g} \mathrm{x} \mathrm{kg}^{-1}$ followed by $\left.2 \mu \mathrm{g} \mathrm{x} \mathrm{kg} \mathrm{h}^{-1}\right)$. The following day, the patient had a stable and regular respiratory pattern at a rate of 24-28 breaths/min and a peripheral capillary oxygen saturation of $>95 \%$. The patient recovered without any evident central nervous system deficits and was discharged on the day after surgery. Subsequently, the patient was subjected to further procedures due to the recurrence of laryngeal papillomatosis. The airway was secured throughout the procedures, using a tracheostomy tube.

\section{Discussion}

Laryngeal papillomatosis frequently occurs in children aged $<5$ years (3). The incidence is $\sim 4.3$ per 100,000 children (2). Due to the exophytic growth properties of papillomatosis, severe laryngeal obstruction may occur in the narrow pediatric airway. In order to relieve airway obstruction, the patient needs to undergo surgical removal of the tumor. The greatest challenge during anesthesia is to secure a patent airway and to ensure adequate ventilation and good surgical field visibility throughout the procedure. A compromised airway is a preexisting risk factor of airway loss during the induction of anesthesia (6). The safest way to prevent a partially obstructed airway from developing into complete obstruction is to keep the patient breathing spontaneously for as long as possible (4).

Inhalation induction of anesthesia is a firmly established technique among the majority of anesthesiologists $(5,7)$. The traditional view is that the technique is safe, since in the case of an obstruction, the volatile agent will no longer be taken up and the patient will be awakened (8); however, as shown in the present case, this technique is not always reliable. Common problems associated with inhalation induction of anesthesia are the following: i) Sevoflurane may irritate the airway and cause laryngospasm and apnea; ii) the airway may collapse; iii) the work of breathing may increase; and iv) a reduction in functional residual capacity $(6,8,9)$. Furthermore, the airway condition of the patients may also contribute to the success or failure of inhalation induction of aneshesia. Li et al (6) found that $60 \%$ of children with grade IV laryngeal obstruction failed to maintain spontaneous breathing following inhalation induction. Similarly, in the present case, during inhalation induction of anesthesia, the child developed complete airway obstruction and suffered a cardiac arrest. Sevoflurane appears to have resulted in further obstruction of the fragile pediatric airway.

With regard to pediatric laryngeal papillomatosis, total intravenous anesthesia with emifentanil and propofol, as well as spontaneous ventilation, has been recommended by a study by Bo et al (9). The majority of children in that study suffered from grade I laryngeal obstruction and 6 patients failed to maintain spontaneous ventilation, although intravenous anesthesia was used. Dexmedetomidine, which does not commonly result in respiratory depression, may be the optimal option for patients with pre-existing severe airway obstruction. Mahmoud et al (10) found that, compared with the use of propofol, dexmedetomidine showed improved preservation of the upper airway muscle tone and minimal airway collapsibility. Furthermore, ketamine has been shown to preserve the hypopharyngeal size in adults (11); however, this compound tends to increase respiratory secretions and induce laryngospasm.

Prior to inhalation induction of anesthesia, a strategy for airway management must have been developed by the surgeon and anesthesiologist, and the two specialists must maintain close communication at all times (12). The anesthesiologist should be prepared for an emergency intubation to address a completely obstructed airway. In addition, the surgeon should be ready to convert the completely obstructed airway into a partially obstructed airway by removing part of the 
papillomatosis tissue, should the emergency intubation fails. Furthermore, experience at a particular skill, such as tracheotomy and cricothyrotomy, is required by both specialists. These skills can be obtained through regular training.

In conclusion, any anesthetic may have a negative effect on patients with a pre-existing airway compromise. Therefore an airway management strategy should be planned prior to the induction of anesthesia, and the surgeon should be prepared to remove part of laryngeal papillomatosis.

\section{References}

1. Derkay CS: Task force on recurrent respiratory papillomas. A preliminary report. Arch Otolaryngol Head Neck Surg 121: 1386-1391, 1995

2. Reeves WC, Ruparelia SS, Swanson KI, Derkay CS, Marcus A and Unger ER: National registry for juvenile-onset recurrent respiratory papillomatosis. Arch Otolaryngol Head Neck Surg 129: 976-982, 2003.

3. Gallagher TQ and Derkay CS: Recurrent respiratory papillomatosis: Update 2008. Curr Opin Otolaryngol Head Neck Surg 16: 536-542, 2008

4. Oshan V and Walker RWM: Anaesthesia for complex airway surgery in children. Contin Educ Anaesth Crit Care Pain 13: 47-51, 2013.
5. Lerman J, Davis PJ, Welborn LG, Orr RJ, Rabb M, Carpenter R, Motoyama E, Hannallah R and Haberkern CM: Induction, recovery, and safety characteristics of sevoflurane in children undergoing ambulatory surgery. A comparison with halothane. Anesthesiology 84: 1332-1340, 1996.

6. Li SQ, Chen JL, Fu HB, Xu J and Chen LH: Airway management in pediatric patients undergoing suspension laryngoscopic surgery for severe laryngeal obstruction caused by papillomatosis. Pediatr Anesth 20: 1084-1091, 2010.

7. Jöhr M and Berger TM: Paediatric anaesthesia and inhalation agents. Best Pract Res Clin Anaesthesiol 19: 501-522, 2005.

8. Patel A: ENT surgery. In: Core Topics in Airway Management. Calder I and Pearce A (eds). 2nd edition. Cambridge University Press, Cambridge, pp227-243, 2011.

9. Bo L, Wang B and Shu SY. Anesthesia management in pediatric patients with laryngeal papillomatosistosis undergoing suspension laryngoscopic surgery and a review of the literature. Int J Pediatr Otorhinolaryngol 75: 1442-1445, 2011.

10. Mahmoud M, Gunter J, Donnelly LF, Wang Y, Nick TG and Sadhasivam S: A comparison of dexmedetomidine with propofol for magnetic resonance imaging sleep studies in children. Anesth Analg 109: 745-753, 2009.

11. Drummond GB: Comparison of sedation with midazolam and ketamine: Effects on airway muscle activity. Br J Anaesth 76: 663-667, 1996.

12. Pearce A and Shaw J: Airway assessment and planning. In: Major Complications of Airway Management in the United Kingdom. Reports and finding. Cook T, Woodall N and Frerk C (eds). The Royal College Of Anesthetists, London, pp135-142, 2011. 